\title{
Successful Endotracheal Intubation Using Suction-Assisted Laryngoscopy Assisted Decontamination Technique and a Head-Down Tilt Position during Massive Regurgitation
}

\author{
Insung Choi, Young Woong Choi, Sang Hyuk Han, Ji Heui Lee \\ Department of Anesthesiology and Pain Medicine, Korea Cancer Center Hospital, Seoul, Korea
}

\begin{abstract}
Some of the challenges in intensive care, anesthesia, and emergency medicine would be airway management in an actively vomiting patient or a bloody airway. We experienced a patient with an excessively full stomach (at least $3 \mathrm{~L}$ ) due to gastric outlet obstruction, although he was scheduled for elective surgery. Despite such circumstances, we successfully intubated him using the suctionassisted laryngoscopy assisted decontamination technique with the patient in a head-down tilt position during massive regurgitation while avoiding resultant pulmonary aspiration.
\end{abstract}

Keywords: Airway management; Decontamination; Head-down tilt; Regurgitation

\section{INTRODUCTION}

Massive regurgitation during airway management presents a challenge in the intensive care unit, operating room, and emergency unit. During such occurrences, resulting aspiration might be associated with serious pulmonary syndromes including acute respiratory distress syndrome, complete respiratory failure, and even cardiopulmonary arrest.

Various techniques that can be used for airway management during massive regurgitation have been developed [1], but due to the scarcity of this event, few publications exist to hold accurate information. Furthermore, supporting evidence is limited because all of them have been studied only in a manikin simulation study for ethical considerations.

In our case, we encountered massive regurgitation during rapid sequence intubation (RSI) in a patient who had a full stomach with gastric outlet obstruction. We successfully intubated him using the suction-assisted laryngoscopy assisted decontamination (SAL$\mathrm{AD})$ technique in a head-down tilt position during massive regurgitation without any complications like a pulmonary aspiration.

\section{CASE REPORT}

The patient in this case was informed of and provided consent regarding the possible publication of his case. A 77-year-old man, diagnosed with gastric cancer, was scheduled for elective radical subtotal gastrectomy. During gastro-endoscopic examination and abdominal computed tomography (CT), a large amount of gastric content was detected in his stomach (Fig. 1A, B). Moreover, a large ulceroinfiltrative mass in his antrum and gastric outlet obstruction was observed. Subsequently, the endoscope could not reach the patient's duodenum.

Prior to the operation, the surgical team neglected the order to insert a nasogastric tube until moments before the patient was transferred to an operating room. Upon arrival in the operating room, we found that the nasogastric tube inserted into the left nostril was misplaced and was stuffed in his right nostril, instead of reaching down into the gastric cavity. The tube was immediately removed. Other than that, the patient had a bulging belly and mentioned to have begun vomiting intermittently several days ago.

Due to our suspicion regarding the full stomach, we altered the (https://creativecommons.org/licenses/by-nc/4.0/). 

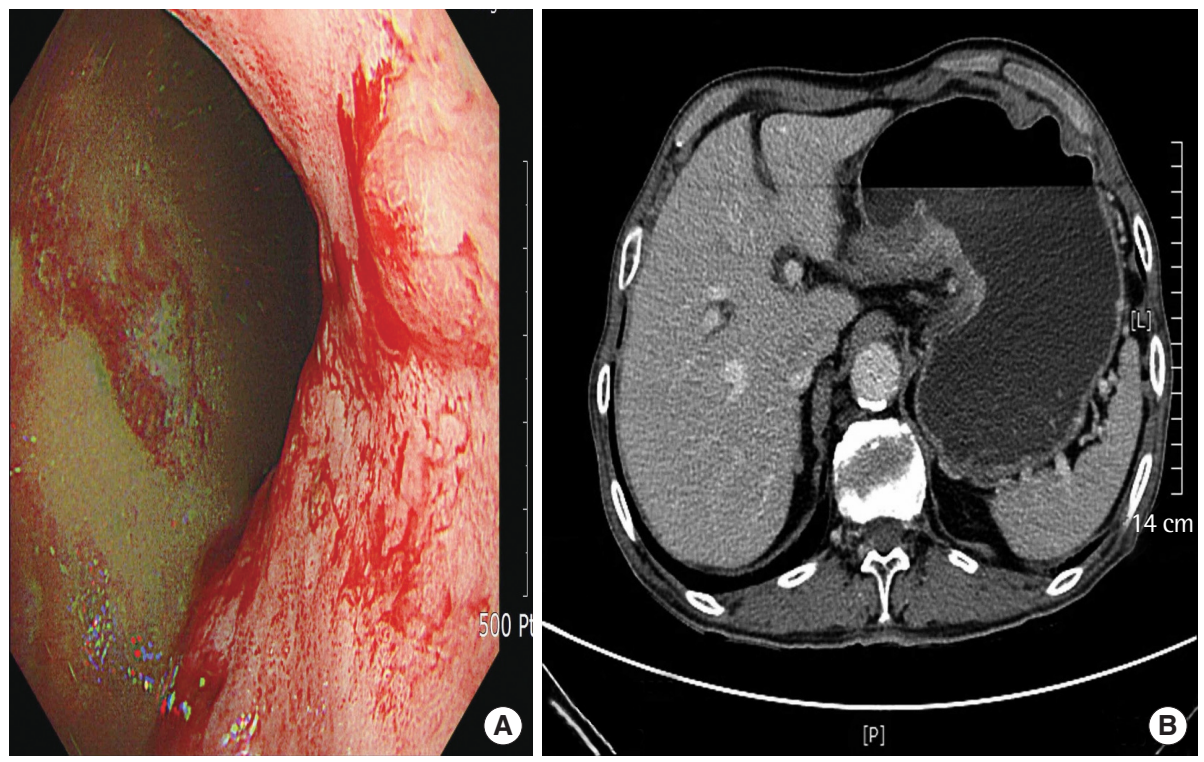

Fig. 1. Gastric endoscopic examination showed large amounts of food materials remaining in the stomach (A) and abdominal computed tomography also revealed severe gastric distension and enormous amounts of gastric content (B).
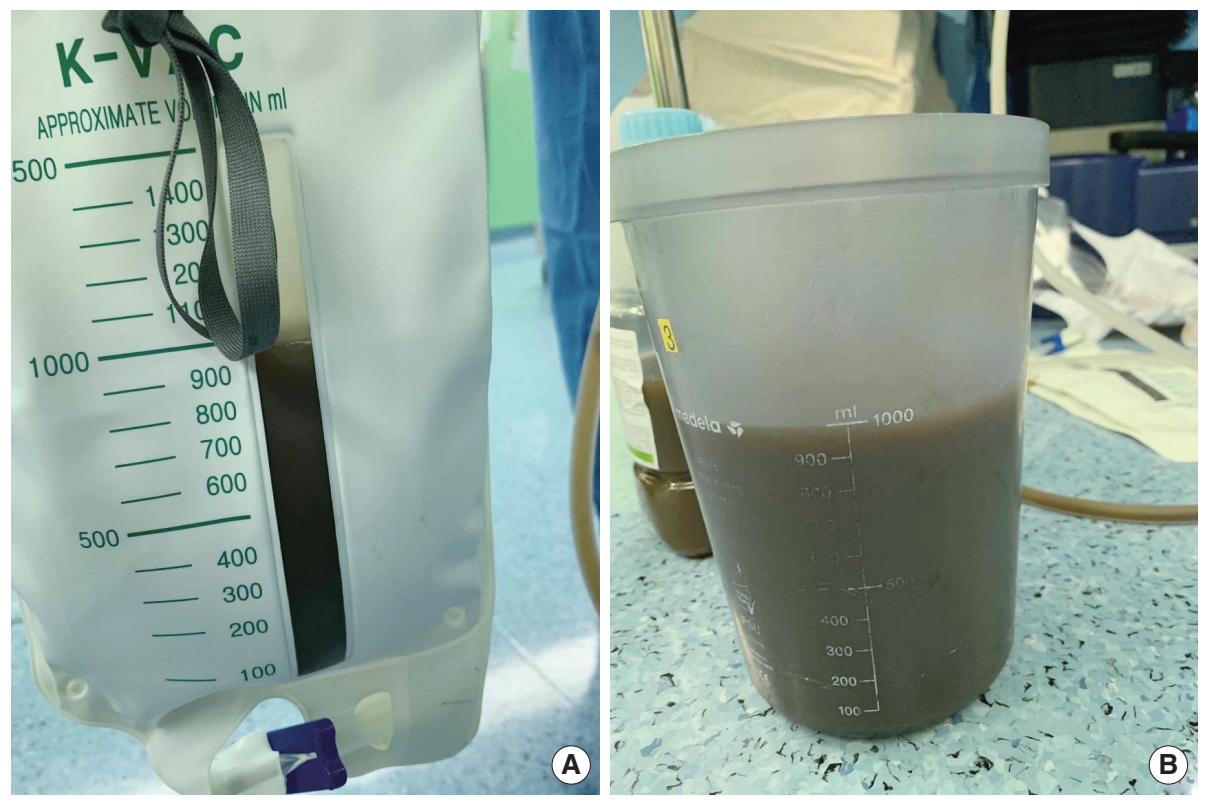

Fig. 2. Large amounts of regurgitated content were observed in the drain bag of the nasogastric tube collecting the aspirated contents immediately after the insertion of a tube $(A)$ and the suction bottle containing the aspirated contents from the oral cavity during intubation (B).

position of the operating table with the patient assuming the over $40^{\circ}$ Trendelenburg position and head-neck extension. After the patient was oxygenated for 10 minutes, we administered $350 \mathrm{mg}$ of thiopental sodium, $60 \mathrm{mg}$ of lidocaine, $1 \mathrm{mg} / \mathrm{kg}$ of rocuronium, $20 \mathrm{mg}$ of esmolol, and $0.5 \mathrm{mg}$ of nicardipine for RSI. Immediately after the patient lost his consciousness, a large amount of bilious gastric content abruptly regurgitated into the oral cavity and out onto the floor. In accordance with The SALAD techniques [2], we inserted a hard suction catheter into the right side of his mouth to suction the regurgitation materials. After inserting a videolaryngoscope, the hard suction catheter was repositioned to the left side of the laryngoscope and suctioned around the esophagus. Afterward, endotracheal intubation was successfully performed through the right side of the laryngoscope. No evidence of pulmonary as- 
piration was observed during endotracheal suction and fiberoptic bronchoscopic examination. Large amounts of gastric content continuously overflowed into the oral cavity and surgical table, and over $1.5 \mathrm{~L}$ of gastric fluid was drained after the insertion of a nasogastric tube (Fig. 2A, B). Even so, a large amount of gastric content still remained within the stomach in the surgical field. During gastrectomy, the patient's oxygen saturation was stably maintained between $98 \%$ and $100 \%$.

After the operation, the patient was extubated and transferred to the postoperative care unit without any signs of dyspnea. $\mathrm{He}$ had no specific symptom and sign of aspiration pneumonia and his oxygen saturation was maintained between $96 \%$ and $100 \%$ in the post-anesthetic care unit and on the ward. His cheat PA checked on a postoperative 1st day showed no active lesion. The patient was discharged on postoperative day 20 without complications related to pulmonary aspiration.

\section{DISCUSSION}

Although the SALAD technique and head-down tilt position have been described in simulation studies, this is the first clinical report about the effectiveness of these techniques for RSI upon massive regurgitation. It is critically important for anesthesiologists and surgeons to have an algorithm for the prevention of aspiration during RSI in patients with gastrointestinal obstruction. Factors contributing to perioperative aspiration pneumonia may include inadequate patient preparation, communication problem, difficulties in surgical techniques, inexperienced providers, and inadequate assistance. In these patients, sufficient nil per os (NPO period and preoperative gastric decompression are essentially required.

Recently, there has been a trend of patient-centric care, and the insertion techniques of various catheters and tubes including nasogastric tubes are delayed to be anesthetized for patients. However, the effort related to the essential decompression of the stomach (enough NPO time, nasogastric tube insertion, etc.) might be neglected even in patients with gastrointestinal obstruction, intraabdominal hypertension, and associated high risk of pulmonary aspiration. Although this case was elective surgery, careless preoperative preparation by the surgical team resulted in a full stomach and massive regurgitation during RSI. The attending surgeon missed the important points related to intra-abdominal hypertension such as serious preoperative vomiting and abdominal disten- sion.

To improve the capability of contaminated airway management, various techniques and some training systems have been developed. The SALAD techniques described by DuCanto et al. [2] involve endotracheal intubation using videolaryngoscopy while simultaneously maintaining oral decontamination using a rigid suction catheter. The suction catheter is placed just below the esophageal orifice to clear the glottic view and prevent additional contamination of the airway. The training course with the SALAD technique significantly improved the performance of soiled airway tracheal intubation: resulting in increased successful intubation rate, shorter intubation time, and improved confidence in RSI [3]. In the manikin study about airway management techniques during massive emesis, there was no difference between the SALAD and intentional esophageal intubation technique in the amount of resultant pulmonary aspiration [1]. However, it showed these techniques might not be the perfect strategies for preventing aspiration during massive regurgitation. The aspiration of regurgitated material could occur during situations in which the rate of massive regurgitation exceeds the capacity of decontamination. We also used the headdown tilt position for the complete prevention of pulmonary aspiration during the SALAD technique.

The classic rapid sequence induction and intubation technique (RSII) for preventing aspiration was discovered approximately 50 years ago, but its main components have remained almost unchanged. Furthermore, until now, the effectiveness of some techniques remains unclear, and a universally accepted RSII method for the complete prevention of pulmonary aspiration is yet to be available. In particular, the ideal position in which RSII is to be conducted remains unclear, and data on the feasibility study about the safest position during RSII are insufficient. According to a German study about the patient's position during RSII in the clinical environment, the preferred position during RSII was the head-up position (84\%), followed by a supine position (13\%), while the headdown position was selected only by $3 \%$ of anesthesiologists [4].

Assuming the head-up position during intubation may decrease the likelihood of regurgitation because of the position's gravitational effect. However, if regurgitation or vomiting occurs due to intragastric pressure exceeding the effect of the head-up position, gastric content may readily enter the trachea and bronchi. Although it has not been extensively analyzed, esophageal pressure during vomiting or regurgitation was hypothesized to be approximately $105 \mathrm{cmH}_{2} \mathrm{O}$ [5], which may exceed the gravitational effects of the 
Choi I, et al. • Intubation during Massive Regurgitation

head-up position.

Recently, according to manikin-based simulating studies, the head-down position and neck extension was observed to minimize airway contamination due to regurgitated contents [6,7]. Takenaka et al. [6] showed that aspiration was dependent on the difference in vertical heights of the tracheal bifurcation, larynx, and mouth. When the corner of the mouth was level with the arytenoid cartilage, gastric contents did not enter the trachea, proving that pulmonary aspiration could be prevented. To completely prevent aspiration, a head-down position of an angle greater than $45^{\circ}$ and $35^{\circ}$ was required in the neutral position and simple extension of head-neck position, respectively. However, in the sniffing position, pulmonary aspiration could not be prevented within the angular range of the head-down tilt position [6]. In our case, we performed a head-down tilt position of over $40^{\circ}$ and a simple extension of the head-neck during RSI. Although the patient had an extremely full stomach with gastric contents of $3 \mathrm{~L}$ or more (including contents in the suction bottle and drain bag of a nasogastric tube, remains in the stomach, and spills on the table and floor), the combined use of a head-down tilt position and neck extension completely prevented the aspiration of these contents. When the tables were tilted to a head-down position, the patients' extremities were tied back by restraining straps to prevent sliding. St. Pierre et al. [7] also found in their manikin simulation study that a $15^{\circ}$ head-down tilt with a Sellick position reduced aspiration but extended the mean intubation times because participants found it more difficult to visualize the vocal cords in the Sellick position than in the familiar sniffing position. They mentioned the possibility of unfamiliarity with the approach in this position to affect such procedures rather than significant anatomical concerns. Therefore, sufficient training regarding the clinical skills of intubation in this position would shorten the intubation time. Following the SALAD technique, we used videolaryngoscopy, which may have helped to see the glottis better. Lewis et al. [8] had also shown that using the videolaryngoscope might improve the glottic view in the situation of difficult intubation and increase the success rate of intubation compared to direct laryngoscopy.

Despite the widespread use of RSI, there are still ongoing controversies concerning the quality of evidence supporting its components and execution of the technique. Cricoid pressure has been the most heated controversy, and in the past 2 decades, some clinicians have questioned its effectiveness and importance. A recent large randomized clinical trial demonstrated that there was no significant difference in the incidence of pulmonary aspiration between the cricoid pressure group and the sham group, with longer intubation time in the cricoid pressure group than in the sham group [9]. In this case, cricoid pressure was not applied during anesthetic induction, because it might not completely prevent aspiration and result in more difficult intubation.

Preoperative nasogastric tube placement has been used to prevent aspiration pneumonia. Mellin-Olsen et al. [10] showed that there was no evidence to support this routine preoperative nasogastric tube insertion, except for patients with gastrointestinal obstruction. This procedure itself may contribute to vomiting and subsequent aspiration in some patients. Hence, the benefits and risks should be carefully considered. We also considered the possibility of vomiting and aspiration during nasogastric tube placement just before anesthetic induction. Furthermore, it was difficult to estimate the amount of gastric content in this case and to determine the delay of the operation for abdominal decompression. Hence, this procedure was not performed until after RSI. Large amounts of gastric content continuously overflowed into the oral cavity even after RSI and the insertion of a nasogastric tube and so, this procedure might not be greatly helpful for the prevention of pulmonary aspiration.

In conclusion, few publications exist that have examined solutions to situations where regurgitation complicates airway management, and we believe the SALAD technique and head-down tilt position may represent an important supplement to airway management during massive regurgitation. We propose that more evidence-based studies about the effectiveness of this combined technique to prevent complications be conducted hereafter.

\section{REFERENCES}

1. Fiore MP, Marmer SL, Steuerwald MT, Thompson RJ, Galgon RE. Three airway management techniques for airway decontamination in massive emesis: a manikin study. West J Emerg Med 2019;20:784-90.

2. DuCanto J, Serrano KD, Thompson RJ. Novel airway training tool that simulates vomiting: suction-assisted laryngoscopy assisted decontamination (SALAD) system. West J Emerg Med 2017;18:117-20.

3. Lin LW, Huang CC, Ong JR, Chong CF, Wu NY, Hung SW. The suctionassisted laryngoscopy assisted decontamination technique toward successful intubation during massive vomiting simulation: a pilot before-after study. Medicine (Baltimore) 2019;98:e17898.

4. Rohsbach C, Wirth S, Lenz K, Priebe H. Survey on the current management of rapid sequence induction in Germany. Minerva Anestesiol 2013; 79:716-26.

5. Brimacombe J, Keller C. Hypopharyngeal seal pressure during projectile vomiting with the ProSeal laryngeal mask airway: a case report and labo- 
ratory study. Can J Anaesth 2006;53:328.

6. Takenaka I, Aoyama K, Iwagaki T. Combining head-neck position and head-down tilt to prevent pulmonary aspiration of gastric contents during induction of anaesthesia: a volunteer and manikin study. Eur J Anaesthesiol 2012;29:380-5.

7. St Pierre M, Krischke F, Luetcke B, Schmidt J. The influence of different patient positions during rapid induction with severe regurgitation on the volume of aspirate and time to intubation: a prospective randomised manikin simulation study. BMC Anesthesiol 2019;19:16.

8. Lewis SR, Butler AR, Parker J, Cook TM, Schofield-Robinson OJ, Smith
AF. Videolaryngoscopy versus direct laryngoscopy for adult patients requiring tracheal intubation: a Cochrane systematic review. Br J Anaesth 2017;119:369-83.

9. Birenbaum A, Hajage D, Roche S, Ntouba A, Eurin M, Cuvillon P, et al. Effect of cricoid pressure compared with a sham procedure in the rapid sequence induction of anesthesia: the IRIS randomized clinical trial. JAMA Surg 2019;154:9-17.

10. Mellin-Olsen J, Fasting S, Gisvold SE. Routine preoperative gastric emptying is seldom indicated: a study of 85,594 anaesthetics with special focus on aspiration pneumonia. Acta Anaesthesiol Scand 1996;40:1184-8. 